\title{
Eficácia e toxicidade do pó comercial de Hoodia gordonii (Masson) Swet ex Decne utilizado no tratamento da obesidade
}

\author{
PEREIRA, C.A. ${ }^{1 *}$; PEREIRA, L.L.S. ${ }^{1}$; CORRÊA, A.D. ${ }^{1}$; RAPOSO, N.R.B. ${ }^{2}$; CASTRO, S.B.R. ${ }^{1}$ \\ ${ }^{1}$ Departamento de Química, Laboratório de Bioquímica, Universidade Federal de Lavras, UFLA/MG, Campus \\ Universitário, Caixa Postal 3037, CEP: 72000-000, Lavras-Brasil ${ }^{2}$ Universidade Federal de Juiz de Fora, UFJF/ \\ MG, Campus Universitário, CEP: 36036-900, Juiz de Fora-Brasil *capfarma@yahoo.com.br
}

\begin{abstract}
RESUMO: A obesidade é atualmente o principal problema de saúde em países desenvolvidos e em desenvolvimento. Diversos recursos terapêuticos têm sido empregados para o tratamento da obesidade destacando-se os fitoterápicos, consagrados pelo conhecimento popular. Nesse contexto, a planta Hoodia gordonii tem despertado atualmente grande interesse mundial, principalmente pelas recentes descobertas e comprovações científicas da inibição do apetite e da sede pelo glicosídeo ativo P57 isolado de espécies da planta. Apesar disso, tais efeitos ainda não foram avaliados e comprovados em amostras comerciais do pó de H. gordonii (PHG), não existindo evidências científicas que garantam a sua eficácia e segurança. Portanto, o objetivo deste trabalho foi realizar ensaios biológicos com ratos para avaliar a atividade farmacológica e a toxicidade de amostras comerciais do pó de H. gordonii. As amostras foram administradas por gavagem em doses equivalentes a 20 vezes as recomendadas para humanos em ratas Wistar durante 4 semanas sendo avaliados os parâmetros indicadores do efeito terapêutico. Após as 4 semanas, os animais foram sacrificados, e amostras de sangue e órgãos foram coletados e submetidos à avaliação dos indicadores metabólicos, endócrinos, hematológicos e histopatológicos. Os resultados demonstraram que para todos os parâmetros avaliados não houve diferenças significativas entre o grupo controle que recebeu somente solução salina estéril e os grupos tratados com PHG indicando que os mesmos, apesar de não apresentarem quaisquer indícios de toxicidade, são incapazes de produzir os supostos efeitos de inibição de apetite e consequente tratamento da obesidade.
\end{abstract}

Palavras-chave: Hoodia, eficácia, toxicidade, obesidade

\begin{abstract}
Efficacy and toxicity of Hoodia gordonii commercial powder used to combat obesity. Obesity is currently the main health problem in developed and developing countries. Several therapeutic methods have been employed for the treatment of obesity, especially herbal medicines, highlighted by popular knowledge. In this context, the plant Hoodia gordonii has currently aroused great interest worldwide, especially for recent discoveries and scientific proof of inhibition of appetite and thirst by the active glycoside P57 isolated from plant species. Nevertheless, such effects have not been evaluated and proven for commercial samples of $H$. gordonii powder (PHG), with no scientific evidence to ensure its effectiveness and safety. Therefore, the aim of this study was to conduct biological tests with rats to evaluate the pharmacological activity and toxicity of commercial samples of $H$. gordonii powder. The samples were administered through gavage, at doses equivalent to 20 times those recommended for humans, in female Wistar rats during 4 weeks for evaluation of the parameters indicative of therapeutic efficacy. After 4 weeks, the animals were sacrificed and blood and organ samples were collected and subjected to the evaluation of metabolic, endocrine, hematological and histopathological indicators. Results showed that for all evaluated parameters, there were no significant differences between the control group that only received sterile saline solution and the groups treated with $\mathrm{PHG}$, indicating that the latter, although presenting no evidence of toxicity, are unable to produce the alleged effects of appetite inhibition and subsequent obesity treatment.
\end{abstract}

Key words: Hoodia, efficacy, toxicity, obesity

Recebido para publicação em 19/07/2010

Aceito para publicação em 28/11/2011

Rev. Bras. Pl. Med., Botucatu, v.14, n.2, p.293-301, 2012. 


\section{INTRODUÇÃO}

O sobrepeso e a obesidade são definidos como o peso que está acima do que é considerado saudável para uma altura específica (CDC, 2008), avaliados por parâmetros como o índice de massa corporal (IMC), circunferência abdominal e outros (OMS, 2010). A obesidade está associada com vários problemas médicos como diabetes mellitus, doenças biliares, osteoartrites, doenças do coração e alguns tipos de câncer (Bray, 2004; SBEM, 2009) e é atualmente o principal problema de saúde em países desenvolvidos e em desenvolvimento (Van Heerden, 2008).

Diversos recursos terapêuticos têm sido empregados para o tratamento da obesidade destacando-se, no entanto, os fitoterápicos e plantas medicinais, consagrados pelo conhecimento popular e utilizados em grande escala pela população como primeiro recurso terapêutico devido ao fácil acesso, baixo custo, não necessidade de prescrição médica e crença na ausência de efeitos colaterais. Levantamentos etnofarmocológicos e etnobotânicos tem demonstrado a utilização de plantas medicinais para o tratamento da obesidade, diabetes e outras doenças metabólicas (Calábria et al., 2008; Cunha Lima et al., 2008). Nesse contexto, a planta Hoodia gordonii tem despertado recentemente grande interesse mundial.

H. gordonii é usada há milhares de anos por tribos do sul do continente africano que atribuem à planta a capacidade de inibir o apetite e a sede (WHO, 2003; Rader et al., 2007; Van Heerden et al., 2007). As recentes descobertas e comprovações de inibição do apetite pelo glicosídeo ativo P57 isolado de espécies de Hoodia (MacLean \& Luo, 2004; Van Heerden et al., 2007) provocaram grande interesse da população, pesquisadores e grandes empresas, existindo atualmente mais de 20 patentes internacionais de produtos comerciais contendo a planta (Van Heerden, 2008).

Estudos envolvendo extratos purificados ou até mesmo os glicosídeos ativos isolados da planta foram realizados (Tulp et al., 2001; MacLean \& Luo, 2004; Van Heerden et al., 2007) comprovando os efeitos farmacológicos de inibição do apetite e da sede e suportando o potencial uso no tratamento da obesidade. No entanto, ensaios biológicos para avaliação dos efeitos a partir da ingestão da planta in natura, sem procedimentos de purificação prévios são raros (Van Heerden, 2008), sendo descrito apenas um trabalho com frangos (Mohlapo et al., 2009). Experimentos semelhantes envolvendo outras espécies como ratos são até o momento inexistentes.

Apesar das evidências científicas da atividade supressora do apetite demonstrada por extratos purificados de $\mathrm{H}$. gordoniique continham o glicosídeo ativo P57, tais efeitos ainda não foram avaliados e comprovados em amostras comerciais do pó de $H$. gordonii. Além disso, ainda não existem evidências científicas que atestem a autenticidade, que sustentem que os mesmos tratam-se de extratos purificados e que garantam a ausência de efeitos deletérios e a segurança do uso. Em função da inexistência de comprovações científicas de sua eficácia e segurança, $H$. gordonii teve a comercialização proibida pela Agência Nacional de Vigilância Sanitária (ANVISA) em fevereiro de 2007 (Anvisa, 2007).

Portanto, neste trabalho foram realizados ensaios biológicos com ratos para avaliar a atividade farmacológica e a toxicidade de amostras comerciais do pó de $H$. gordonii, decorrentes da ingestão diária e contínua do produto. Além disso, foi investigada a influência da ingestão do pó comercial sobre os níveis sanguíneos dos hormônios leptina e insulina, envolvidos na regulação neuroendócrina do apetite e da saciedade.

\section{MATERIAL E MÉTODO}

A avaliação da eficácia e toxicidade das amostras comerciais do pó de Hoodia gordonii foi realizada por meio de ensaios biológicos com ratos, conduzidos no Laboratório de Biofarmácia da Faculdade de Farmácia e Bioquímica da Universidade Federal de Juiz de Fora (FFB/UFJF) e no Laboratório de Neurociências do Departamento e Instituto de Psiquiatria, Faculdade de Medicina da Universidade de São Paulo (USP).

As leituras espectrométricas das análises enzimáticas foram realizadas com os equipamentos: Sistema Delfia - Wallac (PerkinElmer) com 4 módulos (1296-026 Delfia Platewash 1296-041, Delfia Plate Dispense 1296-043, Delfia Dispenser unit 1234, Delfia Fluorometer) e Leitora de Microplacas Spectracount Packard.

Para as análises de glicose, colesterol total, colesterol HDL, triglicérides e Gama-GT foram utilizados kits comerciais Labtest ${ }^{\circledR}$ (Brasil). Para análise de leptina foi empregado kit enzimático Elisa Millipore ${ }^{\circledR}$ (Missouri, EUA) e para insulina, kit AutoDELFIA ${ }^{\circledR}$ (PerkinElmer do Brasil).

Duas amostras comerciais do pó de Hoodia gordonii (denominadas $\mathrm{HA} \mathrm{e} \mathrm{HB}$ ) foram adquiridas em farmácias de manipulação dos municípios de Lavras e Juiz de Fora, ambos em Minas Gerais, acompanhadas de laudos de análises de controle de qualidade realizadas por fornecedores de matérias-primas nacionais, porém de procedência informada da China.

Foram utilizadas no experimento 30 ratas Wistar (Rattus norvegicus) com idade aproximada de 1 mês e peso entre 80 e $100 \mathrm{~g}$, adquiridas junto ao Centro de Bioterismo do Instituto de Ciências Biológicas da Universidade Federal de Minas Gerais

Rev. Bras. Pl. Med., Botucatu, v.14, n.2, p.293-301, 2012. 
(CEBIO/ICB/UFMG). Previamente ao início dos ensaios, os animais passaram por período de 96 horas de ambientação às condições experimentais que envolveram temperatura variando entre $22^{\circ} \mathrm{C}$ a $26^{\circ} \mathrm{C}$, umidade relativa do ar entre $80 \%$ e $90 \%$, ritmo de luz e penumbra num fotoperíodo de 12/12 horas, recebendo ração própria para a espécie (Nuvilab CR1 ${ }^{\circledR}$ ) e água ad libitum, em ambiente exclusivo para roedores e com baixo nível de ruído externo. Durante todo o experimento os animais permaneceram em gaiolas de polietileno $(60 \times 50 \times 22 \mathrm{~cm})$ com cama de maravalha, sempre em número máximo de 10 animais por gaiola. Os cuidados de higienização e alimentação foram diários e realizados por tratador único.

O projeto foi submetido à avaliação prévia da Comissão de Bioética da Pró-Reitoria de Pesquisa da Universidade Federal de Lavras, sendo a seguir considerado aprovado sob o protocolo № 047/2009.

Foi realizado ensaio biológico durante 28 dias para avaliação da toxicidade sub-crônica das amostras de PHG em animais no Laboratório de Biofarmácia da Faculdade de Farmácia e Bioquímica (FFB) da Universidade Federal de Juiz de Fora (UFJF). No planejamento e execução da pesquisa foram obedecidas as recomendações do Colégio Brasileiro de Experimentação Animal (COBEA) e a resolução RE n. 90 da ANVISA de 16 de março de 2004 que determina a publicação do Guia para a realização de estudos de toxidade pré-clínica de fitoterápicos.

Cada amostra foi administrada diariamente, diluída em solução salina estéril por gavagem nos animais do respectivo grupo tratado em doses equivalentes a 20 vezes a dose recomendada para humanos.

Para o cálculo da dose foram considerados a dose máxima sugerida para humanos $(1.600 \mathrm{mg}$ adulto $^{-1} 70 \mathrm{~kg} \mathrm{dia}^{-1}$ ), a dose máxima aproximada correspondente para o animal $\left(2,5 \mathrm{mg}^{2}\right.$ animal $^{-1} 100 \mathrm{~g}$ $\left.\mathrm{dia}^{-1}\right)$, o volume máximo recomendado para 0 procedimento de gavagem $(1 \mathrm{~mL})$ e a máxima solubilidade dos pós no volume recomendado de solução salina estéril. A partir desses cálculos foi selecionada a dose correspondente a 20 vezes a dose recomendada para humanos, equivalente a $0,5 \mathrm{mg} \mathrm{g}^{-1}$ peso corporal do animal. Para tanto, foram preparadas diariamente soluções das amostras HA e HB na concentração de $100 \mathrm{mg} \mathrm{mL}^{-1}$, cujos volumes foram calculados de acordo com o peso total dos animais do grupo. Desta forma, a cada animal foi administrado por gavagem um volume de solução da amostra correspondente à dose calculada em função do peso corporal aferido no dia. O mesmo procedimento foi realizado para o grupo controle ao qual foi administrada apenas solução salina estéril.

Após o período de adaptação os animais foram pesados e distribuídos em 3 grupos de 10 animais cada um, discriminados de acordo com o tratamento a ser administrado por gavagem: grupo 1 (controle), solução salina estéril; grupo 2, vinte vezes a dose usual para humanos em $\mathrm{mg} \mathrm{g}^{-1}$ peso corporal da amostra HA e Grupo 3, vinte vezes a dose usual para humanos em $\mathrm{mg} \mathrm{g}^{-1}$ peso corporal da amostra HB. A administração foi realizada diariamente por gavagem utilizando cânula de aço inoxidável e seringa, esterilizadas e no mesmo horário e pelo mesmo indivíduo, respeitando o volume máximo de $1 \mathrm{~mL}$. Os pesos dos animais bem como os consumos de ração e água (diferença entre a quantidade oferecida e a quantidade restante depois de 24 horas) foram anotados diariamente para avaliação de parâmetros como ganho de peso, curva de crescimento e inibição do apetite e da sede.

No último dia os animais foram submetidos a jejum e restrição hídrica nas 12 horas anteriores ao sacrifício, o qual foi realizado com anestesia prévia em ambiente fechado saturado com vapor de éter etílico, seguido por deslocamento cervical, incisão ventral e exsanguinação por coleta de sangue do coração. Após o sacrifício foi realizada também a necropsia, com retirada de órgãos internos (coração, fígado e rins,) para preparação de lâminas histológicas.

Os resultados foram submetidos à análise de variância utilizando o software SISVAR (Ferreira, 2000).

O peso corporal de cada animal foi aferido em balança de precisão e anotado diariamente durante todo o experimento para a avaliação da curva de crescimento e desenvolvimento dos animais, ganho de peso, bem como parâmetro de análise do efeito sobre $o$ apetite.

Utilizando o sangue coletado do coração, foram realizadas dosagens laboratoriais para avaliação de parâmetros bioquímicos ou metabólicos (glicose, colesterol total, colesterol HDL, triglicérides e GamaGT), hematológicos (esfregaço sanguíneo), e endócrinos (insulina e leptina). Para as análises de parâmetros bioquímicos e endócrinos foram utilizados kits enzimáticos comerciais enquanto que os esfregaços sanguineos foram analisados em microscópio e a seguir confirmados por analisador automático. Todas as análises foram realizadas no Laboratório de Biofarmácia da Faculdade de Farmácia e Bioquímica da Universidade Federal de Juiz de Fora (FFB/UFJF), à exceção da análise de insulina que foi realizada no Laboratório de Neurociências da Faculdade de Medicina da Universidade de São Paulo (USP).

Para as análises histopatológicas de órgãos internos foram preparadas lâminas histológicas a partir de fragmentos dos órgãos retirados na necropsia. No processamento histológico, os órgãos foram seccionados em pequenos fragmentos, fixados com formalina tamponada nas primeiras 24 horas e após, armazenados em álcool $70 \%$ para conservação até o

Rev. Bras. PI. Med., Botucatu, v.14, n.2, p.293-301, 2012. 
procedimento de inclusão. Posteriormente, os fragmentos foram processados para inclusão em blocos de parafina, que a seguir foram submetidos à microtomia, resultando cortes de cerca de $3 \mu \mathrm{m}$ de espessura. Os cortes obtidos foram então corados pelo método da hematoxilina-eosina (HE) e montados em lâminas/lamínulas de vidro para avaliação histopatológica com microscópio óptico.

\section{RESULTADO E DISCUSSÃO}

$\mathrm{Na}$ Tabela 1 são apresentados os resultados de alguns indicadores bioquímicos dosados no sangue dos animais em estudo.

A determinação da glicemia é o parâmetro inicial para detecção de alterações e distúrbios do metabolismo glucídico como o diabetes tipo 2, normalmente associado à obesidade (Motta, 2009; SBEM, 2009). Os níveis de glicose encontrados revelaram não haver diferenças significativas entre os grupos tratados (HA e HB) e o grupo controle, indicando que as amostras de PHG não exercem qualquer efeito na glicemia (Teste $F$ não significativo).
A dosagem sanguínea de lipídeos e enzimas hepáticas fornece importantes parâmetros na determinação da segurança de ingredientes funcionais ou de produtos finais derivados de plantas submetidos a ensaios de toxicidade (Patel et al., 2008). Os níveis elevados de colesterol total e triglicérides estão diretamente associados ao prognóstico de risco de aterosclerose coronariana, doença cardíaca isquêmica e infarto, enquanto o colesterol HDL possui efeito protetor contra esses distúrbios. A gama-GT por sua vez, tem aplicação principal no estudo de desordens hepato-biliares, apresentando maior especificidade que outras enzimas hepáticas como a fosfatase alcalina e as transaminases (Motta, 2009).

Os resultados das dosagens de colesterol total, triglicérides e colesterol HDL foram estatisticamente iguais para os grupos tratados e controle e indicam que o PHG não interfere no metabolismo lipídico, sendo desprovido de quaisquer efeitos relacionados a alterações das taxas de lipídeos sanguíneos, sejam eles benéficos ou deletérios. Em adição, os resultados de gama-GT sugerem positivamente que o PHG não possui efeito

TABELA1. Níveis sanguíneos médios ${ }^{1}$ de glicose e lipídeos e atividade de gama-GT nos três grupos de tratamento.

\begin{tabular}{lccccc}
\hline Grupo & $\begin{array}{c}\text { Glicose } \\
\left(\mathbf{m g ~ d L}^{-1}\right)\end{array}$ & $\begin{array}{c}\text { Triglicérides } \\
\left(\mathbf{m g ~ d L}^{-1}\right)\end{array}$ & $\begin{array}{c}\text { Colesterol } \\
\left(\mathbf{m g ~ d L}^{-1}\right)\end{array}$ & $\begin{array}{c}\mathbf{H D L} \\
\left(\mathbf{m g ~ d L}^{-1}\right)\end{array}$ & $\begin{array}{c}\text { Gama-GT } \\
\left(\mathbf{U ~ L}^{-1}\right)\end{array}$ \\
\hline Controle & $135,9 \pm 25,1$ & $76,7 \pm 14,4$ & $95,9 \pm 12,5$ & $61,8 \pm 4,9$ & $95,7 \pm 2,5$ \\
HA & $134,27 \pm 20,1$ & $70,1 \pm 13,3$ & $83,9 \pm 13,8$ & $61,1 \pm 5,4$ & $98,2 \pm 4,5$ \\
HB & $138,3 \pm 25,2$ & $75,1 \pm 15,1$ & $84,4 \pm 11,9$ & $56,4 \pm 5,7$ & $96,7 \pm 1,7$ \\
\hline
\end{tabular}

${ }^{1}$ Dados são a média \pm desvio padrão $\quad{ }^{2} U \mathrm{~L}^{-1}=$ atividade da enzima em unidades, proporcionais à quantidade do produto $\mathrm{p}$-nitroanilina liberado na reação enzimática e lido em $405 \mathrm{~nm}$, por litro de amostra.

hepatotóxico (Teste $\mathrm{F}$ não significativo).

$\mathrm{Na}$ Tabela 2, estão apresentados os níveis sanguíneos dos hormônios leptina e insulina envolvidos na regulação neuroendócrina da ingestão de alimentos. Observou-se níveis sanguíneos estatisticamente iguais para os dois hormônios entre os grupos estudados (Teste F não significativo).

A leptina é produzida principalmente no tecido adiposo que atua no sistema nervoso central (hipotálamo) promovendo a regulação da função neuroendócrina e o metabolismo da glicose e gorduras (Friedman \& Halaas, 1998; Reseland et al., 2001). A ingestão alimentar e o gasto energético são regulados pela leptina, sendo os níveis circulantes proporcionais à massa de gordura corporal total (Frederich et al., 1995; Pelleymounter et al., 1995; Morton et al., 2006).

Alguns autores (Spanswick et al., 1997; Bays, 2004) demonstraram que a leptina inibe neurônios hipotalâmicos por ativação de canais de potássio ATP-dependentes, o mesmo local relatado por MacLean \& Luo (2004) como sendo o alvo da
TABELA 2. Níveis sanguíneos médios ${ }^{1}$ de leptina e insulina nos três grupos de tratamento.

\begin{tabular}{lcc}
\hline Grupo & $\begin{array}{c}\text { Leptina } \\
\left(\mathbf{n g ~ m L} \mathbf{~}^{-1}\right)\end{array}$ & $\begin{array}{c}\text { Insulina } \\
\left(\mu \mathrm{U} \mathbf{~ m L}^{-1}\right)\end{array}$ \\
\hline Controle & $1,68 \pm 0,25$ & $16,69 \pm 1,23$ \\
$\mathrm{HA}$ & $1,59 \pm 0,21$ & $17,56 \pm 1,11$ \\
$\mathrm{HB}$ & $1,61 \pm 0,22$ & $17,04 \pm 1,14$ \\
\hline
\end{tabular}

${ }^{1}$ Dados são a média \pm desvio padrão.

ação inibidora do apetite pelo glicosídeo ativo P57 presente em $\mathrm{H}$. gordonii.

Baixos níveis de leptina induzem alterações metabólicas no sentido de preservar as reservas de energia existentes e ao mesmo tempo provocam alterações cerebrais de tal forma que a aquisição e consumo de alimentos torna-se extremamente importante (Berthoud \& Morrison, 2008). Em oposição, níveis aumentados podem indicar uma 
leptina-resistência que é descrita como um estado similar à insulino-resistência, no qual a elevada leptinemia ocorre simultaneamente à hiperfagia e à obesidade (Myers et al., 2008).

Os resultados de leptina encontrados não confirmaram a hipótese da influência do PHG sobre o hormônio, evidenciado pelos níveis sanguíneos inalterados em relação ao grupo controle. No entanto, deve-se ressaltar que no presente estudo foram utilizadas amostras comerciais da planta de autenticidade não confirmada, e não o glicosídeo P57 purificado utilizado em outros estudos, devido a não disponibilidade do produto purificado. Esse fato limita uma comparação mais ampla dos resultados desse estudo com os demais. Apesar disso, como o propósito do trabalho foi analisar os produtos disponibilizados para a população como inibidores de apetite, tais resultados são pertinentes e relevantes.

A insulina por sua vez, está envolvida no metabolismo glucídico, tomando parte principalmente na redução dos níveis glicêmicos. Entretanto, a exemplo da leptina, foi atribuída à insulina nos últimos anos, atividade no sistema nervoso central graças a descoberta de receptores para o hormônio no cérebro (Hallschmid \& Schultes, 2009). Agentes que aumentem a sensibilidade e diminuam os níveis sanguíneos de insulina podem ser promissores no tratamento da obesidade, particularmente em pacientes com diabetes tipo 2 (Bays, 2004). Contudo, os resultados de insulina obtidos nesse trabalho indicam que o pó comercial de $H$. gordonii não afeta os níveis sanguíneos do hormônio.

A partir de uma gota de sangue de cada animal foi realizado esfregaço sanguíneo para contagem de células. Os resultados são apresentados na Tabela 3.

Os resultados das contagens celulares revelaram não haver diferenças significativas entre os grupos analisados (Teste $F$ não significativo), evidenciando apenas o padrão leucocitário normal de inversão entre o número de linfócitos e neutrófilos em relação a humanos e típico de ratos.

$\mathrm{Na}$ Figura 1 pode-se observar a curva de crescimento dos animais tratados e controle semanalmente durante todo o período do experimento.

TABELA 3. Contagem de glóbulos brancos* para os três grupos de tratamento.

\begin{tabular}{lccc}
\hline & \multicolumn{3}{c}{ Grupo } \\
\cline { 2 - 4 } & Controle & HA & HB \\
\hline Neutrófilos & $14,0 \pm 3,4$ & $15,0 \pm 3,6$ & $14,0 \pm 4,1$ \\
Linfócitos & $79,0 \pm 6,9$ & $78,0 \pm 6,1$ & $81,0 \pm 4,8$ \\
Monócitos & $6,0 \pm 1,3$ & $6,0 \pm 1,8$ & $5,0 \pm 1,6$ \\
Eosinófilos & $1,0 \pm 0,7$ & $1,0 \pm 0,9$ & $1,0 \pm 0,5$ \\
Basófilos & - & - & - \\
\hline
\end{tabular}

* Os dados representam as médias \pm desvio padrão de cada tipo celular avaliados em esfregaço sanguíneo com microscópio óptico e confirmados por analisador automático.

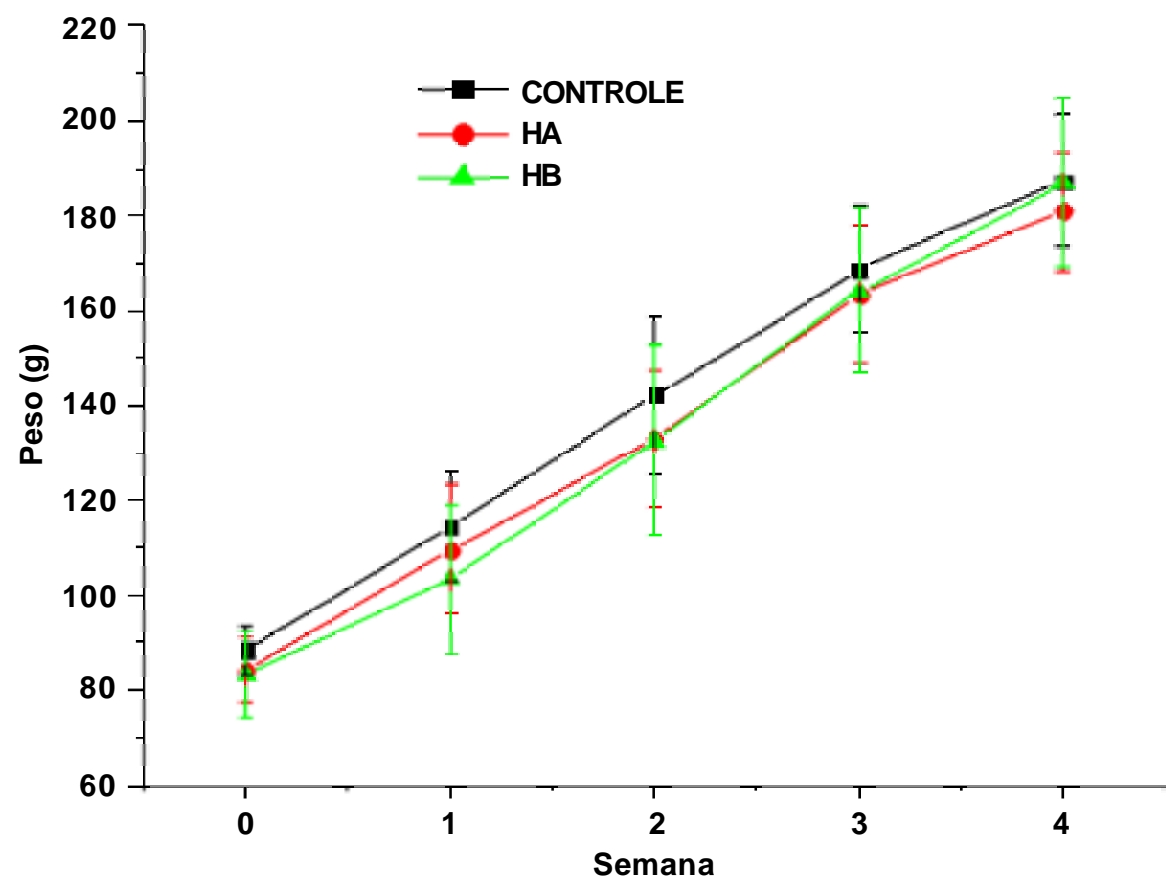

FIGURA 1. Curva de crescimento dos ratos durante os 28 dias de experimento de cada tratamento. Os pontos representam as médias \pm desvio padrão do peso médio dos grupos em cada semana do experimento. Não houve diferença significativa entre os grupos em todas as semanas avaliadas. 
Verificou-se que a tendência de crescimento entre os grupos foi semelhante, indicando que o PHG não influenciou no ganho de peso dos animais tratados (Teste $\mathrm{F}$ não significativo).

Na Figura 2 são apresentados os valores de ganho de peso médio dos grupos, calculados pela diferença entre o peso inicial e o peso final dos animais.

Os resultados de ganho de peso médio durante o período do experimento revelaram que não houve diferenças significativas (Teste $F$ não significativo) entre os grupos tratados (92,92 g para HA e 93,80 g para HB) e o grupo controle $(94,71 \mathrm{~g})$, evidenciando que as amostras de PHG não afetaram o apetite $e$, consequentemente, o ganho de peso dos animais tratados.

Resultados de diminuição do peso foram encontrados em ratos tratados com extratos purificados contendo glicosídeos de $\mathrm{H}$. gordonii (Tulp et al., 2001; 2002; Van Heerden et al., 2007). No entanto, a administração de pó de $H$. gordonii, adicionada a ração em concentrações de 0 a $500 \mathrm{mg}$ animal $^{-1} \mathrm{dia}^{-1}$ não demonstrou efeito na diminuição do peso em frangos (Mohlapo et al., 2009). Neste estudo, porém, o teor relatado do glicosídeo ativo P57 (13,95 $\mathrm{mg} \mathrm{kg}^{-1}$ de matéria seca) indica que na dose máxima de $H$. gordoniiutilizada $(500 \mathrm{mg})$ havia cerca de $7 \times 10^{-6} \mathrm{~g}$ deste, valor cerca de dez vezes menor ( $7 \times 10^{-5} \mathrm{~g}$ de $\left.\mathrm{P} 57\right)$ que o necessário para inibir 50\% do apetite de ratos quando injetado diretamente no cérebro, segundo MacLean \& Luo (2004), e que pode explicar em parte o resultado encontrado.

Levantamentos etnofarmacológicos sugerem que a $H$. gordonii é capaz de inibir a fome e a sede (Bruyns, 1993; WHO, 2003; Van Heerden, 2008). No intuito de investigar esses efeitos, foram avaliados os consumos médios de ração e água dos grupos durante todo período do experimento (Tabela 4).

Verificou-se que não houve diferenças significativas (Teste $F$ não significativo) nos consumos médios de ração e água entre os grupos tratados e controle, indicando que mesmo em doses equivalentes a 20 vezes a dose recomenda para humanos as amostras comerciais do pó de $H$. gordonii não são capazes de provocar qualquer inibição do apetite ou da sede.

Adicionalmente, observaram-se na Figura 3 as relações de consumo de ração e água por peso médio dos animais. Os resultados refletem percentuais de relação consumo de ração/peso animal $(11,68,12,06$ e 11,81\%) e relação consumo de água/peso animal (18,0, 18,4 e 17,3\%), estatisticamente iguais (Teste $F$ não significativo) entre os grupos controle, HA e HB, respectivamente.

Ensaios biológicos utilizando preparações semi-purificadas de quatro espécies de Hoodia adicionadas na ração de ratos Wistar durante 3 semanas, demonstraram que houve supressão do apetite dos animais tratados, sendo necessários aproximadamente $2,5 \mathrm{mg}$ de $H$. gordonii g peso animal ${ }^{-1}$ para obtenção da $\mathrm{DE}_{50}$ (Dose efetiva capaz de provocar a atividade esperada em $50 \%$ dos animais testados) (Tulp et al., 2001). Resultados semelhantes foram obtidos com ratos obesos tratados durante 3 semanas com um homogeneizado obtido de Hoodia previamente desidratada (Tulp et al., 2002). Extratos purificados de $H$. gordonii e $H$. pilifera contendo glicosídeos ativos administrados por gavagem em ratas Wistar em proporções de 6,25 a $50,00 \mathrm{mg} \mathrm{kg}^{-1}$ dia $^{-1}$ durante oito dias foram efetivos na diminuição do consumo de ração (Van Heerden et al., 2007). Adicionalmente,

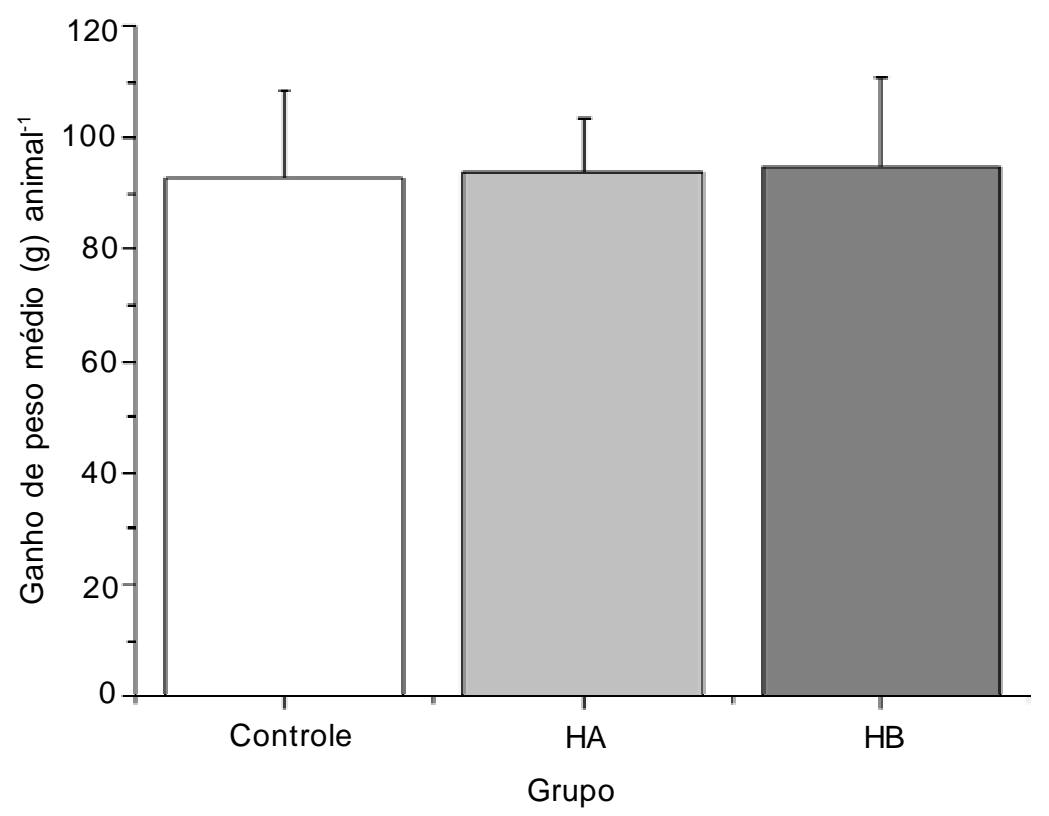

FIGURA 2. Ganho de peso médio durante os 28 dias de experimento para os três grupos de tratamento. 
TABELA 4. Consumo médio por animal de ração e água por dia durante os 28 dias de experimento para os três grupos de tratamento.

\begin{tabular}{|c|c|c|}
\hline Grupo & $\begin{array}{l}\text { Consumo médio } \\
\left.\text { de ração ( } \text { d dia }^{-1}\right)\end{array}$ & $\begin{array}{l}\text { Consumo médio }{ }^{1} \\
\text { de água }\left(\mathrm{mL} \mathrm{dia} \mathrm{di}^{-1}\right)\end{array}$ \\
\hline Controle & $15,34 \pm 2,51$ & $24,7 \pm 7,8$ \\
\hline $\mathrm{HA}$ & $16,09 \pm 2,50$ & $26,4 \pm 7,5$ \\
\hline $\mathrm{HB}$ & $15,33 \pm 3,47$ & $22,4 \pm 7,3$ \\
\hline
\end{tabular}

${ }^{1}$ Consumo médio por animal.

foi demonstrado em ratos que injeções intracerebroventriculares do glicosídeo ativo P57 isolado de $\mathrm{H}$. gordonii, reduziram a ingestão alimentar entre 40 e $60 \%$ nas 24 horas subsequentes à administração (MacLean \& Luo, 2004).

Por outro lado, a suplementação de ração de frangos de corte com pó de $H$. gordonii, em proporções de 0 a $500 \mathrm{mg}_{\text {animal }}{ }^{-1}$ dia $^{-1}$ durante 30 dias não revelou qualquer alteração no consumo de ração dos grupos tratados em relação ao grupo controle (Mohlapo et al., 2009). Em humanos, estudos iniciais não publicados pela companhia Phytopharm, revelaram que após 15 dias de ingestão de $H$. gordonii, 19 indivíduos com obesidade mórbida tiveram a ingestão calórica reduzida em quase $50 \%$ (MacLean \& Luo, 2004).

Os resultados encontrados para as duas amostras comerciais de PHG não revelaram nenhum efeito na inibição do apetite e da sede nos animais submetidos ao tratamento com doses equivalentes a
20 vezes aquelas recomendadas para humanos. Entretanto, convém ressaltar que diferentemente da maioria dos autores citados, nesse estudo, as amostras utilizadas não foram submetidas a qualquer procedimento de purificação para obtenção de princípios ativos, tratando-se apenas de pós comercializados como "extratos da planta". Essas descobertas permitem questionamentos quanto à autenticidade dos pós comerciais da planta e quanto à presença e quantidade nesses pós dos glicosídeos ativos, cujos efeitos já foram cientificamente comprovados. Tais preocupações tem sido demonstradas por diversos autores (Rader et al., 2007; Dall'Acqua \& Innocenti, 2007; Avula et al., 2008; Van Heerden, 2008) que revelam apreensão quanto a grande possibilidade de fraudes diante do crescente interesse mundial em contraposição à pequena disponibilidade de plantas cultivadas.

$\mathrm{Na}$ Figura 4 são apresentadas imagens microscópicas capturadas dos órgãos submetidos aos processamentos histológicos. Não foram observadas alterações macroscópicas, à dissecção, nos órgãos analisados, isto é, coração, fígado e rins. Os processamentos histológicos ocorreram de forma similar em todos os grupos. Para cada órgão/animal/ grupo, foi realizada uma fotomicrografia, de área mais significativa (aumento de 100x). Não foi observada nenhuma lesão ou alteração microscópica significativa (Hematoxilina-eosina, $\mathrm{HE}$ ) entre os tecidos animais avaliados dos diversos grupos estudados (Fotomicrografias a,b e c).

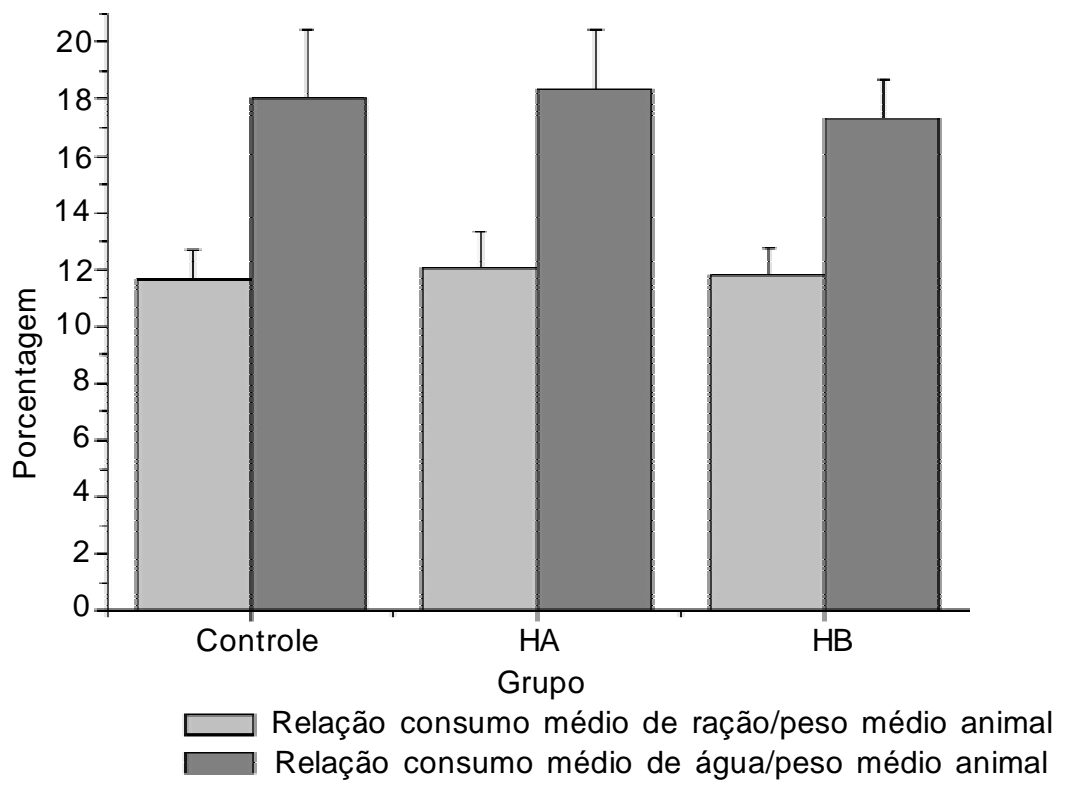

FIGURA 3. Relações do consumo de ração/peso animal e consumo de água/peso animal durante os 28 dias de experimento para os três grupos de tratamento. 

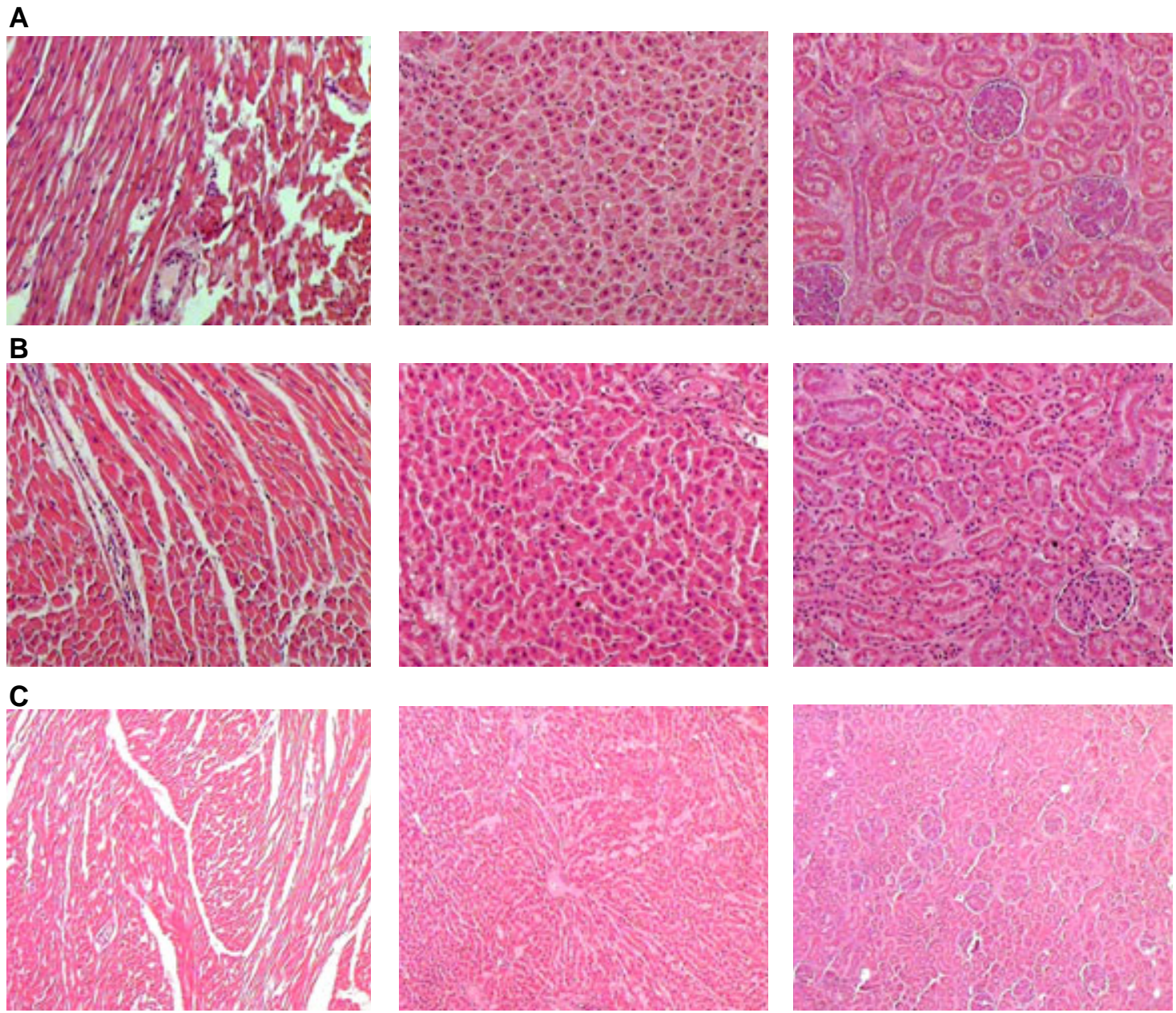

FIGURA 4. Fotomicrografias de coração, fígado e rins (da esquerda para direita), utilizando coloração HE com aumento de 100x: A. controle; B. HA; C. HB.

\section{CONCLUSÃO}

As duas amostras comerciais do pó de $H$. gordoniiadministradas em ratas em doses equivalentes a 20 vezes as recomendadas para humanos não provocam qualquer alteração nos parâmetros metabólicos (glicose, colesterol total, colesterol HDL, triglicérides e gama-GT), hematológicos (esfregaço sanguíneo) e endócrinos (leptina e insulina), além de não exercerem nenhuma influência no apetite e na sede dos animais tratados. Adicionalmente, a avaliação de toxicidade revela que as amostras são desprovidas de efeitos deletérios, considerando o período de 28 dias de tratamento.

\section{AGRADECIMENTO}

Nossos agradecimentos à CAPES pela bolsa de doutorado e à FAPEMIG pelo apoio financeiro ao projeto.

\section{REFERÊNCIA}

ANVISA - Agência Nacional de Vigilância Sanitária. Resolução-RDC no 48, de 16 de março de 2004. Disponível em: <http://www.anvisa.gov.br>. Acesso em: 23 set. 2009.

AVULA, B. et al. A rapid method for chemical fingerprint analysis of Hoodia species, related genera, and dietary supplements using UPLC-UV-MS. Journal of Pharmaceutical and Biomedical Analysis, v.48, p.72231, 2008.

BAYS, H.E. Current and investigational antiobesity agents and obesity therapeutic treatment targets. Obesity Research, v.12, n.8, p.1197-211, 2004.

BERTHOUD, H.R.; MORRISON, C. The brain, appetite, and obesity. Annual Review of Psychology, v.59, p.5592, 2008.

BRAY, G.A. Medical consequences of obesity. The Journal of Clinical Endocrinology \& Metabolism, v.89, p.2583-9, 2004.

BRUYNS, P.V. New combinations in hoodia and lavrania (Asclepiadaceae - Stepelieae). South African Journal of Botany, v.59, n.3, p.342,1993. 
CALÁBRIA, L. et al. Levantamento etnobotânico e etnofarmacológico de plantas medicinais em Indianópolis, Minas Gerais, Brasil. Revista Brasileira de Plantas Medicinais, v.10, n.1, p.49-63, 2008. CENTERS FOR DISEASE CONTROLAND PREVENTION (CDC), USA. Disponível em: <http://www.cdc.gov/ccdphp/ dnpa/obesity/defining.htm>. Acesso em: 10 jan. 2010. CUNHA LIMA, S.T. et al. Levantamento da flora medicinal usada no tratamento de doenças metabólicas em Salvador, BA- Brasil. Revista Brasileira de Plantas Medicinais, v.10, n.4, p.83-9, 2008.

DALL'ACQUA, S.; INNOCENTI, G. Steroidal glyxosides from Hoodia gordonii. Steroids, v.72, p.559-68, 2007. FERREIRA, D.F. Análises estatísticas por meio do SISVAR para windows versão 4.0. In: REUNIÃO BRASILEIRA DA SOCIEDADE INTERNACIONAL DE BIOMETRIA. 45., 2000, São Carlos. Programa e Resumo... São Carlos: UFSCar, 2000. p.235.

FREDERICH, R.C. et al. Leptin levels reflect body lipid content in mice: evidence for diet-induced resistance to leptin action. Nature, v.1, p.1311-4, 1995.

FRIEDMAN, J.M.; HALAAS, J.L. Leptin and the regulation of body weight in mammals. Nature, v.395, n.22, p.76370, 1998.

HALLSCHMID, M.; SCHULTES, B. Central nervous insulin resistance: a promising target in the treatment of metabolic and cognitive disorders? Diabetologia, v.52, p.2264-9, 2009.

MacLEAN, D.B.; LUO, L. Increased ATP content/ production in the hypothalamus may be a signal for energy-sensing of satiety: studies of the anorectic mechanism of a plant steroidal glycoside. Brain Research, v.1020, p.1-11, 2004.

MOHLAPO, T.D. et al. Effect of Hoodia gordonii meal supplementation at finisher stage on productivity and carcass characteristics of Ross 308 broiler chickens. Tropical Animal Health and Production, v.41, p.1591-6, 2009.

MORTON, G.J. et al. Central nervous system control of food intake and body weight. Nature, v.443, p.289-95, 2006. MOTTA, V.M. Bioquímica clínica para o laboratório: princípios e interpretações. Rio de Janeiro: MedBook,
2009, 400p.

MYERS, MG.; COWLEY, MA.; MUNZBER, G.H. Mechanisms of leptin action and leptin resistance. Annual Review of Psychology, v.70, p.537-56, 2008. ORGANIZAÇÃO MUNDIAL DA SAÚDE (OMS). Disponível em: <http://www.who.int>. Acesso em: 12 jan. 2010.

PATEL, C. et al. Safety assessment of pomegranate fruit extract: acute and subchronic toxicity studies. Food and Chemical Toxicology, v.46, p.2728-35, 2008.

PELLEYMOUNTER, M.A. et al. Effects of the obese gene product on body weight regulation in ob/ob mice. Science, v.269, p.540-3, 1995

RADER, J.I. et al. Recent studies on selected botanical dietary supplement ingredients. Analytical and Bioanalytical Chemistry, v.389, p.27-35, 2007. RESELAND, J. E. et al. Effect of long-term changes in diet and exercise on plasma leptin concentrations. American Journal of Clinical Nutrition, v.73, n.2, p.240-5, 2001

SOCIEDADE BRASILEIRA DE ENDOCRINOLOGIA E METABOLOGIA (SBEM). Disponível em: <http://www. endocrino.org.br/busca/obesidade>. Acesso em: 15 jan. 2010

SPANSWICK, D. et al. Leptin inhibits hypothalamic neurons by activation of ATP-sensitive potassium channels. Nature, v.390, n.4, p.521-5, 1997.

TULP, O.L. et al. Effect of Hoodia plant on food intake and body weight in lean and obese $L A / N t u l / /-c p$ rats. FASEB Journal, v.15, p.A404, 2001.

TULP, O.L. et al. Effect of Hoodia plant on weight loss in congenic obeseLA/Ntul//-cp rats. FASEB Journal, v.16, n.4, p.A654, 2002.

VAN HEERDEN, F.R. Hoodia gordonii: A natural appetite suppressant. Journal of Ethnopharmacology, v.119, p.434-7, 2008.

VAN HEERDEN, F.R. et al. An appetite suppressant from Hoodia species. Phytochemistry, v.68, p.254553, 2007.

WORLD HEALTH ORGANIZATION - WHO. Bulletin of the World Health Organization, v.81, n.5, p.382, 2003. 